\title{
New Understanding of Fanconi Anemia Signaling Network upon Studying FANCD2
}

\author{
Kanani Hokutan ${ }^{1,2}$, Jin-Hee Kim ${ }^{1}$, Sudong Zhan ${ }^{1}$, Jun Zhang ${ }^{3}$ and Peiwen Fei ${ }^{1,2}$ * \\ ${ }^{1}$ University of Hawaii Cancer Center, Hawaii, US \\ ${ }^{2}$ Graduate Program of Molecular Biosciences and Bioengineering, University of Hawaii, Hawaii, USA \\ ${ }^{3}$ Department of Pathology and Laboratory Medicine, Mayo Clinic Foundation, USA
}

*Corresponding author: Peiwen Fei, MD PhD, University of Hawaii Cancer Center, 701 Ilalo Street, Honolulu, HI 96813, USA.

To Cite This Article: Peiwen Fei, New Understanding of Fanconi Anemia Signaling Network upon Studying FANCD2. Am J Biomed Sci \& Res. 2020 - 7(2). AJBSR.MS.ID.001130. DOI: 10.34297/AJBSR.2020.07.001130.

Received: 眥 January 25, 2020; Published: 眥 February 05, 2020

\begin{abstract}
The Fanconi Anemia (FA) pathway is activated upon replication stress and DNA damage. With the accumulated studies, this pathway has emerged as a fundamental signaling network to defend genome stability. The Fanconi Anemia complementation group D2 protein (FANCD2) sits at the center of the pathway, orchestrating many players to prevent our genome from going awry, leading to diseases including cancer. Here, we highlight recent advances in our understanding of FA signaling, emphasizing on a rarely known form of FANCD2, FANCD2-V2.
\end{abstract}

\section{Fanconi Anemia}

Fanconi anemia is a rare genetic disease, characterized by developmental and physical abnormalities, bone marrow failure and increased cancer susceptibility [1-9]. The majority of children with FA are likely to develop myelodysplastic syndrome or acute myeloid leukemia (AML), while adults are predisposed to head and neck squamous cell carcinoma, hepatocellular carcinoma, gynecological, and gastrointestinal cancers $[1-3,5,6,8,10,11]$. FA is caused by germline mutations in any of the 22 FA genes (FANCA/B/C/D1/ $\mathrm{D} 2 / \mathrm{E} / \mathrm{F} / \mathrm{G} / \mathrm{I} / \mathrm{J} / \mathrm{L} / \mathrm{M} / \mathrm{N} / 0 / \mathrm{P} / \mathrm{Q} / \mathrm{R} / \mathrm{S} / \mathrm{T} / \mathrm{U} / \mathrm{V} / \mathrm{W}$ ). FA gene products maintain genomic integrity and participate in a common DNA repair pathway- the FA/BRCA pathway $[2,4,12,13]$. Proteins involved are specialized more in resolving DNA interstrand cross-links (ICL), a lesion that blocks both DNA replication and transcription $[2,5,14]$. Patients suffering from FA display high frequencies of chromosomal abnormalities and are hypersensitive to DNA crosslinking agents (i.e. mitomycin C, cisplatin, diepoxybutane) $[1,3,5,6,8]$. If DNA is not properly repaired it can lead to genomic instability, apoptosis, senescence and tumorigenesis [4,7].

\section{FA Signaling}

Proteins implicated in the FA/BRCA pathway coordinate nearly all known DNA repair mechanisms in order to resolve ICLs [1-5].
Given accumulated studies showing much crosstalk between FA and other important DNA damage repair proteins, FA signaling pathway emerges as a significant signaling network, responding to a variety of genotoxic stresses. This network is activated upon replication stress or DNA damage. FANCM is a DNA translocase and forms a protein complex with FAAP24 (FA-associated protein 24) and others. FANCM-FAAP24 recognizes stalled replication forks due to the ICL $[1,2]$. Histone-fold containing kinetochore protein MHF1MHF2 allows for the stable association of FANCM to chromatin [5]. ATR checkpoint kinase phosphorylates FANCM proceeding its recruitment to the site of damage [3]. Eight FA proteins assemble and form the FA core complex (FANCA, B, C, E, F, G, L, M) in addition to FAAP100, FAAP20, and FAAP24 to act as E3 ubiquitin ligase. Similarly, to FANCM, FANCD2 and FANCI are phosphorylated in an ATR-dependent manner. FANCT acts as an E2 together with the FA complex E3 responsible for monoubiquitinating FANCD2 and FANCI at Lys 561 and Lys 523, respectively [15]. The monoubiquitination of FANCD2-FANCDI (ID2) complex is a key step, representing FA signaling network activation. Therefore, FANCD2 must be tightly regulated to maintain DNA repair. The ID2 complex moves to the DNA lesion and recruits nucleases (FANCP/SLX4 and ERCC1/ FANCQ/XPF) to unhook the ICL $[2,16]$. The incision is converted to 
a double strand break (DSB) and translesion synthesis (TLS) allows for continued replication of the leading strand, while the lagging strand is mediated by homologous recombination (HR) $[3,5,14,17$ 20]. However, it remains largely unclear as to how each individual DNA damage repair mechanism works in concert to fully repair the damage within the expanded FA signaling network, including many FA and non-FA proteins that are yet to be identified.

\section{FA Signaling and Cancer}

Therefore, if the FA pathway is impaired due to mutations in involved genes, DNA remains damaged, promoting genotoxic stress, genomic instability, and tumorigenesis [3,21,22]. For instance, unresolved ICLs cause DNA breakage and chromosomal rearrangement, leading to cancer development [4]. Mutations or epigenetic silencing of FA genes are found in breast, ovarian and pancreatic cancers, and heterozygous germline mutations in BRCA2/FANCD1, BRIP1/FANCJ, PALB2/FANCN, RAD51C/FANCO, and BRCA1/FANCS are important cancer risk alleles [6,12,23-25]. Recently, researchers performed whole genome sequencing on three patients presented with three up to five primary cancers. Interestingly, the varied genes in each patient are part of the FA pathway [26]. FA gene defects are found in a variety of human cancers. Of the FA genes, FANCA has the highest mutation rate and is associated with AML, pancreatic, cervical, oral and prostate cancers [1,27]. An elevated FANCA expression determines a worse outcome for patients with chronic lymphocytic leukemia (CLL) and is due to a reduction of p53 genes, p21 and $\Delta$ Np73 [28]. Another group found FANCA amplification correlates with reduced progression-free survival in head and neck squamous cell carcinoma after radiotherapy [27]. FANCC has the next highest mutation rate and is similarly associated with pancreatic, cervical and oral cancers in addition to breast cancer [1,6,29]. Like any other FA gene, mutations in FANCD2 gene are involved in a variety of malignancies, including testicular and esophageal squamous cell carcinoma [1]. However, its importance is far beyond cancer implications, as FANCD2 sits at the center of the FA signaling network, and its activation/monoubiquitination represents the activation of FA signaling. With our recent studies on FANCD2, the previous unrecognized variant of FANCD2, FANCD2-V2 appears to be a more potent tumor suppressor than the commonly known form of FANCD2, FANCD2-V1. This review will focus on our current knowledge of FANCD2-V2. The related studies will aid in further understanding the roles that FANCD2 and/or FA signaling play in the development of human cancer and other diseases.

\section{Overlooked FANCD2 Variant}

Previously, our lab reported the first study on an alternate variant of FANCD2, named FANCD2-V2 (NCBI RefSeq accession\#NM_033084.4) [30]. We refer to the long-known form as FANCD2-V1. FANCD2-V2's cDNA is 60 bp longer than FANCD2-V1, encoding a 1471 amino acid (aa) transcript compared to FANCD2-V1 at 1451 aa. Both variants share 1427 aa at the $\mathrm{N}$-terminus, resulting in $95 \%$ aa identity. We checked the expression of both FANCD2 variants in human lung tumor and matched normal samples. Interestingly, V2/V1 (ratio of V2 to V1) expression is higher in normal/non-malignant tissue compared to malignant tissues. We further assessed a panel of normal (CRL-1790, HEK293, WI-38) and tumor (HEK293T, PA-1, U2OS, HCT116, HT-29, RKO, LoVo) cell lines, which demonstrate the same trend.

In examining FANCD2 gene structure, we found a proximal and distal polyadenylation site (PAS). When the proximal PAS is used the FANCD2-V2 transcript loses the last intron; however, this intron is incorporated in the FANCD2-V1 transcript. We learned through RNA polymerase II ChIP that more DNA fragments were pulled down at the distal PAS in malignant cells compared to nonmalignant cells. U2 snRNP RNA Immunoprecipitation (RIP) showed SF3A1 antibody, a component of U2 snRNP, pulled down fragments representing the last FANCD2-V1 intron with greater binding in malignant cells than non-malignant cells. This finding suggests FANCD2-V1 transcripts are more prevalent in malignant cells. Additionally, human bladder and ovarian cancer samples present an increased V2/V1 expression in lower stage cancers. These data demonstrate FANCD2-V2 could be a more potent tumor suppressor as its expression is high in non-malignant cells and tissues.

Studies propose more than $50 \%$ of mammalian genes have multiple PAS [30]. How are sites of polyadenylation determined? A possible cause is DNA methylation. We recently reported, treating HEK293 with DNA methyltransferases reduced FANCD2-V2 expression and DNA methylation intensity near the proximal APS by methylated DNA immunoprecipitation (MeDIP) [31]. Our findings were validated with publicly available datasets (TCGA) in lung, ovarian, kidney, endometrial, colon and breast cancer, displaying an elevated methylated distal to methylated proximal APS ratio (Me-D/Me-P) is associated with malignancy. A high level of DNA methylation in the distal APS promotes FANCD2-V1 expression. Conversely, a high level of DNA methylation in the proximal APS results in FANCD2-V2 expression. To this end, we identified how two forms of FANCD2 are differentially expressed and connected with human tumorigenesis. However, it is largely unknown as to which traits in contributing to the functions are common or different between them. Simply, we would also ask; do additional FANCD2 variants exist? How are they expressed and/or functioning.

\section{Conclusion and Prospective}

In this short review, we aimed to update our understanding of FA signaling network with a focus on the center player, FANCD2. We described a recent recognized form of FANCD2, FANCD2-V2. This variant is expressed higher in non-malignant cell lines and tissues comparing to the corresponding malignant ones, due to proximal APS usage. These data suggest the long-known form FANCD2-V1 
may be rather oncogenic compared to FANCD2-V2, which is more tumor suppressive. Of note, another group identified an alternatively splice isoform of FANCE, FANCE $\Delta 4$, which is expressed in breast cancer patients without BRCA1/2 mutations [32]. FANCE $\Delta 4$ blocked cells into G2/M phase after MMC treatment, reduced cell survival, and restricted FANCD2 and FANCI from monoubiquitination. Perhaps additional FA genes rely on alternative splicing for proper expression and gene regulation. More studies are needed to define the function(s) of alternative isoforms as wild-type protein function maybe disrupted or the isoform could gain new functions. These studies will allow for further characterization of FA genes and a deeper understanding of the FA signaling network.

\section{Acknowledgements}

We thank previous lab members and research support from NIH and University of Hawaii Cancer Center.

\section{References}

1. Nepal M, Che R, Zhang J, Ma C, Fei P (2017) Fanconi Anemia Signaling and Cancer. Trends Cancer 3(12): 840-856.

1. Jo U, Kim H (2015) Exploiting the Fanconi Anemia Pathway for Targeted Anti-Cancer Therapy. Mol Cells 38(8): 669-676.

2. Bhattacharjee S, Nandi S (2017) DNA damage response and cancer therapeutics through the lens of the Fanconi Anemia DNA repair pathway. Cell Commun Signal 15(1): 41.

3. Sumpter R Jr, Levine B (2017) Emerging functions of the Fanconi anemia pathway at a glance. J Cell Sci 130(16): 2657-2662.

4. Kim H, D'Andrea AD (2012) Regulation of DNA cross-link repair by the Fanconi anemia/BRCA pathway. Genes Dev 26(13): 1393-1408.

5. Taniguchi T, D'Andrea AD (2006) Molecular pathogenesis of Fanconi anemia: recent progress. Blood 107(11): 4223-4233.

6. Zhang T, Du W, Wilson AF, Namekawa SH, Andreassen PR, et al. (2017) Fancd2 in vivo interaction network reveals a non-canonical role in mitochondrial function. Sci Rep 7: 45626.

7. Park E, Kim H, Kim JM, Primack B, Vidal Cardenas S, et al. (2013) FANCD2 activates transcription of TAp63 and suppresses tumorigenesis. Mol Cell 50(6): 908-918.

8. Che R, Zhang J, Nepal M, Han B, Fei P (2018) Multifaceted Fanconi Anemia Signaling. Trends Genet 34(3): 171-183.

9. Farshid AM, Haanstra JR, de Looper HW, van Strien PM, Verhagen Oldenampsen J (2015) ICL- induced miR139-3p and miR199a-3p have opposite roles in hematopoietic cell expansion and leukemic transformation. Blood 125(25): 3937-3948.

10. Freie B, Li X, Ciccone SL, Nawa K, Cooper S, et al. (2003) Fanconi anemia type $\mathrm{C}$ and p53 cooperate in apoptosis and tumorigenesis. Blood 102(12): 4146-4152.

11. Sumpter R Jr, Sirasanagandla S, Fernandez AF, Wei Y, Dong X, et al. (2016) Fanconi Anemia Proteins Function in Mitophagy and Immunity. Cell 165(4): 867-881.

12. Ma C, Nepal M, Kim JH, Fan P, Fei P (2019) A new look at molecular biology of breast cancer. Cancer Biol Ther 20(1): 1-5.

13. Huang Y, Li L (2013) DNA crosslinking damage and cancer - a tale of friend and foe. Transl Cancer Res 2(3): 144-154.

14. van Twest S, Murphy VJ, Hodson C, Tan W, Swuec P, et al. (2017)
Mechanism of Ubiquitination and Deubiquitination in the Fanconi Anemia Pathway. Mol Cell 65(2): 247-259.

15. Liang Z, Liang F, Teng Y, Chen X, Liu J, et al. (2019) Binding of FANCIFANCD2 Complex to RNA and R-Loops Stimulates Robust FANCD2 Monoubiquitination. Cell Rep 26(3): 564-572.

16. Schlacher K, Wu H, Jasin M (2012) A distinct replication fork protection pathway connects Fanconi anemia tumor suppressors to RAD51BRCA1/2. Cancer Cell 22(1): 106-116.

17. Rickman KA, Lach FP, Abhyankar A, Donovan FX, Sanborn EM, et al. (2015) Deficiency of UBE2T, the E2 Ubiquitin Ligase Necessary for FANCD2 and FANCI Ubiquitination, Causes FA-T Subtype of Fanconi Anemia. Cell Rep 12(1): 35-41.

18. Federico MB, Campodonico P, Paviolo NS, Gottifredi V (2018) Beyond interstrand crosslinks repair: contribution of FANCD2 and other Fanconi Anemia proteins to the replication of DNA. Mutat Res 808: 83-92.

19. Nepal M, Che R, Ma C, Zhang J, Fei P (2017) FANCD2 and DNA Damage. Int J Mol Sci 18(8): 1804.

20. Nepal M, Ma Ch, Xie G, Jia W, Fei P (2018) Fanconi Anemia complementation group C protein in metabolic disorders. Aging. 10(6): 1506-1522.

21. Panneerselvam J, Xie G, Che R, Su M, Zhang J, et al. (2016) Distinct Metabolic Signature of Human Bladder Cancer Cells Carrying an Impaired Fanconi Anemia Tumor-Suppressor Signaling Pathway. J Proteome Res 15(4): 1333-1341.

22. Stoepker C, Ameziane N, van der Lelij P, Kooi IE, Oostra AB, et al. (2015) Defects in the Fanconi Anemia Pathway and Chromatid Cohesion in Head and Neck Cancer. Cancer Res 75(17): 3543-3553.

23. Hess CJ, Ameziane N, Schuurhuis GJ, Errami A, Denkers F, et al. (2008) Hypermethylation of the FANCC and FANCL promoter regions in sporadic acute leukaemia. Cell Oncol 30(4): 299-306.

24. Catucci I, Osorio A, Arver B, Neidhardt G, Bogliolo M, et al. (2018) Individuals with FANCM biallelic mutations do not develop Fanconi anemia, but show risk for breast cancer, chemotherapy toxicity and may display chromosome fragility. Genet Med 20(4): 452-457.

25. Wang L, Wang H, Wang T, Liu J, Chen W, et al. (2019) Analysis of polymorphisms in genes associated with the FA/BRCA pathway in three patients with multiple primary malignant neoplasms. Artif Cells Nanomed Biotechnol 47(1): 1101-1112.

26. Hess J, Unger K, Orth M, Schotz U, Schuttrumpf L, et al. (2017) Genomic amplification of Fanconi anemia complementation group A (FancA) in head and neck squamous cell carcinoma (HNSCC): Cellular mechanisms of radioresistance and clinical relevance. Cancer Lett 386: 87-99.

27. Bravo Navas S, Yanez L, Romon I, Pipaon C (2019) Elevated FANCA expression determines a worse prognosis in chronic lymphocytic leukemia and interferes with p53 function. FASEB J 33 (9): 10477-10489.

28. Kottemann MC, Smogorzewska A (2013) Fanconi anaemia and the repair of Watson and Crick DNA crosslinks. Nature 493(432): 356-363.

29. Han BSY, Zhang P, Panneerselvam J, Che R, Zhang J, etal. (2017) Overlooked FANCD2 variant encodes a promising, potent tumor suppressor, and alternative polyadenyaltion contributes to its expression. Oncotarget 8(4): 22490-22500.

30. Ma C, Wang Z, Nepal M, Hokutan K, Zhang J, et al. (2018) DNA methylation at the vicinity of the proximal polyadenylation site in FANCD2 gene involves human malignancy. Cell Cycle 17(17): 2204-2206.

31. Bouffard F, Plourde K, Belanger S, Ouellette G, Labrie Y, et al. (2015) Analysis of a FANCE Splice Isoform in Regard to DNA Repair. J Mol Biol 427(19): 3056-3073. 